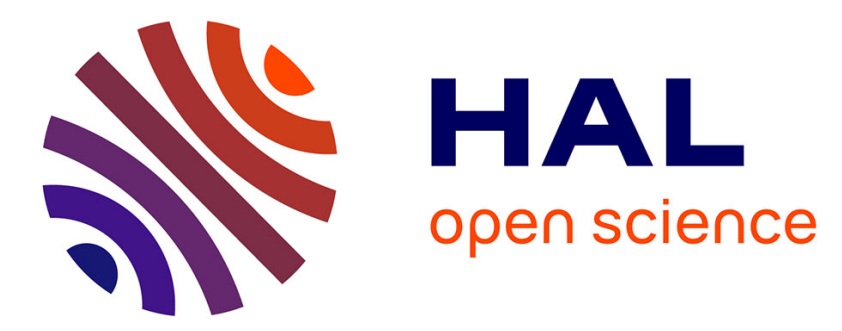

\title{
Selective muscle contraction during plantarflexion is incompatible with maximal voluntary torque assessment
}

Nicolas A Turpin, Antony Costes, David Villeger, Bruno Watier

\section{To cite this version:}

Nicolas A Turpin, Antony Costes, David Villeger, Bruno Watier. Selective muscle contraction during plantarflexion is incompatible with maximal voluntary torque assessment. European Journal of Applied Physiology, 2014, 114 (8), pp.1667-1677. 10.1007/s00421-014-2900-3 . hal-01662999

\section{HAL Id: hal-01662999 \\ https://hal.laas.fr/hal-01662999}

Submitted on 13 Dec 2017

HAL is a multi-disciplinary open access archive for the deposit and dissemination of scientific research documents, whether they are published or not. The documents may come from teaching and research institutions in France or abroad, or from public or private research centers.
L'archive ouverte pluridisciplinaire HAL, est destinée au dépôt et à la diffusion de documents scientifiques de niveau recherche, publiés ou non, émanant des établissements d'enseignement et de recherche français ou étrangers, des laboratoires publics ou privés. 
Nicolas A. Turpin, Antony Costes, David Villeger and Bruno Watier

N. A. T urpin $\cdot$ A. Costes $\cdot$ D. Villeger $\cdot$ B. Watier

UPS, PRISSMH, University of T oulouse, 118 route de Narbonne, 31062 T oulouse Cedex 9, France

B. Watier

CNRS LAAS, 7 Avenue du colonel Roche, 31077 Toulouse, France

B. Watier $\left(^{*}\right)$

PRISSMH-EA 4561-F2SMH, Pôle Sport, 118 route de Narbonne, 31062 T oulouse Cedex 9, Francee-mail:

bruno.watier@univ-tlse3.fr 
Objective. Large variations in maximal voluntary torque are reported in the literature during

21 isometric plantarflexion contractions. We propose that these differences, which could reach $40 \%$ across similar studies, could be explained by differences in the instructions provided, and notably by instructions as to favoring or not multi-joint contractions.

Method. Sixteen participants were placed on an isokinetic ergometer in 3 different positions,

supine, prone and seated, with the ankle in the neutral position, and instructed to create maximal force on the footplate by conforming with instructions that favored either isolated (ISOL) or multi-joint (ALL) isometric contractions. Torque, foot kinematics and the electromyographic activity of seven muscles of the lower limb have been recorded.

Results. Joint torques were greater in ALL compared to ISOL $(p<.05)$ with gains of $43.5[25.4$ $170.6] \%, 42.5[1.4194 .6] \%$ and $15.3[9.371 .9] \%$ in the supine, prone and seated position, respectively (values are given as median [range]). The results suggested that forces created by muscles that do not span over the ankle joint significantly influenced the measured joint torque, notably in the seated position. Nevertheless, the observed gains in torque were associated with greater plantarflexor muscles activation, showing that the ISOL condition may have induced a form of inhibition of these muscles.

Conclusions. The results of this study suggest that using isolated contractions, hence constrained testing protocols, cannot provide optimal conditions for MVC testing, notably for plantarflexor muscles, which seem to be extremely sensitive to such constrained conditions.

Keywords. EMG, maximal voluntary contraction; plantarflexion; multi-joint contraction; concurrent activation 


\section{list of abbreviations}

ALL: multi-joint contractions condition

ANOVA: analysis of variance

CR: center of rotation

EMG: electromyographic signal

EMGmax: maximal electromyographic (EMG) value obtained over all conditions

GM: gastrocnemius medialis

Gmax: gluteus maximus

ISOL: isolated contractions condition

MVC: Maximal voluntary contraction

RF: rectus femoris

SCoRE: Symmetrical Centre of Rotation Estimation method

SCS: segment coordinate system

SD: standard deviation

Sol: soleus

ST: semi tendinosus

TA: tibialis anterior

VL: vastus lateralis 


\section{Introduction}

Maximal voluntary contraction (MVC) torque is an important measure to evaluate mechanical properties of the muscle and their progress with physical training (Klass et al. 2008; Van Cutsem et al. 1998) or in rehabilitation, to assess the evolution of musculoskeletal diseases and to quantify the beneficial effects of different therapeutic strategies (Moraux et al. 2013; McNeil et al. 2007). MVC at the ankle joint is especially critical to consider due to the important role of the plantarflexor and dorsiflexor muscles in maintaining balance and avoiding fall (Horak et al. 1989). Still, MVCs evaluation requires several precautions to be taken, because mechanical and neural factors could greatly influence torque output. Therefore, the present study will focus on isometric plantarflexion MVCs.

Regarding mechanical factors, even though ergometers have proven to be reliable instrument per se (Drouin et al. 2004), many biases are known to affect measurements, such as, (i) gravitational effects, (ii) inertial effects, (iii) compliance of the ergometer moment arm or deformation of the footplate and fasteners compliance, or (iv) misalignments between the axis of rotation of the ergometer relative to that of the joint (Arampatzis et al. 2007; Herzog 1988; Deslandes et al. 2008), that, moreover, represents only an approximation of the actual functional axis of rotation of the joints (Ramos and Knapik 1978; Hicks 1953). In the isometric case, gravitational effects are easily eliminated and the inertial effects are supposed to be negligible (Deslandes et al. 2008). Compliance involves movement of the segment relative to the moment arm of the ergometer, and implies that muscles MVCs cannot be evaluated at the exact intended position. Adjustments can nevertheless be easily performed to correct positional changes observed when the muscles go from the passive to the active state (De Ruiter et al. 2008). Misalignment, on the other hand, is particularly critical for the evaluation of plantarflexion as compared to other group of muscles. 


$$
\tau_{\text {ankle }}=\frac{r_{\text {ankle }}}{r_{\text {dynamometer }}} \tau_{\text {dynamometer }}
$$

with $\tau_{\bullet}$ the torque at either the ankle or the dynamometer axis, and $r_{\bullet}$ the moment arm of the reaction force to either the ankle or the dynamometer. This relation could be rewritten as:

$$
\tau_{\text {ankle }}=\left(1+\frac{\Delta r}{r_{\text {dynamometer }}}\right) \tau_{\text {dynamometer }} ; \Delta r=r_{\text {ankle }}-r_{\text {dynamometer }}
$$

highlighting that, for a given misalignment $\Delta r$ (order of magnitude $=1 \mathrm{~cm}$ ), the bias is lower for knee extension testing (with large $r_{\text {dynamometer }}$ relative to $\Delta r ; \quad r_{\text {dynamometer }} \approx 30-40 \mathrm{~cm}$, (Arampatzis et al. 2004; Deslandes et al. 2008) than for plantarflexion testing $\left(r_{\text {dynamometer }} \approx\right.$ $17 \mathrm{~cm}$, assuming that the forces on the footplate act at the level of the metatarsophalangeal joint of the big toe, Van Cutsem et al. 1998). In addition, with misalignment, a moment arm is created between the ankle joint and the axis of rotation of the ergometer, and the reactions forces at the level of the ankle joint can thus create a torque on the footplate without any torque on the foot. Moreover, these forces can be easily manipulated by the participant using forces created by muscles not crossing the ankle joint (e.g., knee or hip extensors), and these accessory muscles can then have a mechanical influence on the measured joint torque.

At least two neural factors should be considered in this juncture: motivation and concurrent activation potentiation (Ebben et al. 2008a; Ebben et al. 2010), also referred to as remote voluntary contraction (Cherry et al. 2010; Ebben et al. 2008b). Motivation is a wellknown confound variable influencing performance which can be controlled following several recommendations (see Gandevia (2001) notably for a review). Concurrent activation potentiation is much less considered and captures the fact that contraction of accessory muscles 
(remote contraction) may increase the maximal activation level of primary movers (Ebben et al. 2008b). This phenomenon is commonly attributed to motor irradiation and/or to an increase in spinal excitability (Ebben 2006). Jaw clenching, Valsalva maneuver and hand gripping have been particularly investigated (see Ebben et al. (2008b) for a review), but muscles from adjacent sites also proves to interact with the primary movers (Barry et al. 2008; Devanne et al. 2002; Kouchtir-Devanne et al. 2012). It is therefore likely that muscles that do not span over the ankle joint have also a neural influence on plantarflexor activity and hence on plantarflexion torque. Since accessory muscles may come into play at both the neural and the mechanical level, the aim of this study was to test the maximal torque produced in plantarflexion using two modalities of instructions aimed at manipulating the degree of involvement of muscles not crossing the ankle joint. Furthermore, the various positioning used in the literature, notably the seated (Moraux et al. 2013; Simoneau et al. 2009), prone (Cresswell et al. 1995; Maganaris 2003) and supine positions (Danneskiold-Samsøe et al. 2009; Simoneau et al. 2007) are likely to favour specific patterns of muscle activity, and thus to influence the results in a different way. Therefore, in this study these three positions have been tested. Offset of the rotation axes, ankle angle deviations and muscle activity have been recorded in order to set apart the neural and mechanical influences of the accessory muscles. 
103 Materials and methods

104

105

106

107

108

109

110

111

112

113

114

115

116

117

118

119

120

121 124 of them 3 tries were given to the participant, resulting in a total of 18 MVCs.

Participants

16 healthy males participated to the study (mass=76.8 $\pm 8.5 \mathrm{~kg}$, range $=[68-92]$; height $=1.77 \pm 0.07 \mathrm{~m}$, range $=[1.62-1.87] ;$ age $=26.9 \pm 6.4$ years, range $=[20-41])$. All of them were informed of the experimental procedures prior to giving their written consent to participate. The experimental design of the study was approved by the local ethical committee and the experiments were conducted in accordance with the Declaration of Helsinki (last modified in 2004).

\section{General procedure}

Ankle torque measurements were performed using an isokinetic dynamometer (Biodex III, Shirley Corporation, NY, USA). The right leg was evaluated in all participants. Participants were equipped of the reflective markers used for kinematic analysis and of the recording electromyographic (EMG) surface electrodes at their arrival at the laboratory (see details in sections Kinematics and Electromyographic acquisition). We first estimated the position of the real center of rotation of the ankle by moving passively the ankle on the dynamometer footplate in a procedure described in section Estimation of the ankle rotation axis and center of rotation. Afterward, participants performed a warm up lasting 5 minutes which consisted of submaximal isometric plantarflexor contractions while seated on the ergometer. Participants were then successively placed in the PRONE, SUPINE or SEATED position in a random order to assess their isometric MVCs. For each of these positions two modalities of instruction were randomly given to the participants. These constitute a total of $3 \times 2=6$ randomized conditions and for each

125 Kinematics 
A motion analysis system (Vicon Motion System, Lake Forest, CA) equipped with 11 infrared cameras recorded the 3-dimensional position of 11 reflexive markers stuck on the participant and on the dynamometer. Markers were positioned on the right side of the body at the level of the external and internal maleolli, calcaneous (posterior point of the heel), 1st and

130 5th Metatarsal Head, fibula's head and tibiale's tuberosity. 4 reflexives markers were placed on

131 the dynamometer such that the mid distance between two of the markers corresponds to the

132 position of the dynamometer axis of rotation and that the two others, placed in a more backward

133 position, allowed to recover the direction of this axis. Kinematic data were recorded at a

134 sampling frequency of $200 \mathrm{~Hz}$. Ankle angle represents the angle between the vector going from

135 the calcaneous to the midpoint between 1st and 5th Metatarsal Head, and the vector going from

136 the midpoint between fibula's head and tibiale's tuberosity to the midpoint between the two

137 maleolli (see Figure 1).

138 Electromyographic acquisition

139 Surface EMG was recorded from 7 muscles located on the right side of the body, namely,

140 tibialis anterior (TA), soleus (Sol), gastrocnemius medialis (GM), vastus lateralis (VL), rectus

141 femoris (RF), semi tendinosus (ST) and gluteus maximus (Gmax). Prior to electrode application,

142 the skin was shaved and cleaned with alcohol to minimize impedance. Pairs of Ag-AgCl disk

143 electrodes of $8 \mathrm{~mm}$ diameter with inter electrode-distances of $2 \mathrm{~cm}$ were placed longitudinally

144 with respect to the underlying muscle fibers arrangement according to the recommendations of

145 Surface EMG for Non-Invasive Assessment of Muscles (SENIAM) (Hermens et al. 2000). The

146 references electrode was placed at the level of the great trochanter. EMG signals were amplified

147 ( $\times 1000)$, digitized (6-400 Hz bandwidth) at a sampling rate of $1 \mathrm{kHz}$ (Biopac System Inc.

148 Goleta, USA), recorded and synchronized using the motion analysis system. 


\section{Conditions of MVC testing and recording}

The ankle joint torque was acquired with the isokinetic dynamometer and digitally synchronized at a sample rate of $1 \mathrm{kHz}$ using the motion analysis system. During MVCs, participants were positioned on the ergometer and securely stabilized by using two crossover shoulder harnesses and a belt across the abdomen. The right foot was strapped securely to the footplate with the ankle fixed at an angle of $90^{\circ}$ i.e., at the neutral position with the sole of the foot perpendicular to the shank, and held in place by a heel block. The axis of the dynamometer was aligned with the anatomical ankle flexion-extension axis, estimated as the line passing through the tips of the maleolli (Wu et al. 2002; Lundberg et al. 1989). A clear start and stop signals were given. Each voluntary contraction lasted approximately 3-4 s and 1 minute of rest were given between each contraction (Todd et al. 2004). Participants received no feedback of their performances during the tests.

\section{Positions}

Three positions were tested, PRONE, SUPINE and SEATED. For PRONE and SUPINE positions the participants were lying on the dynamometer chair with the hip and the knee fixed at an angle of $0^{\circ}$ (=full extension for both). In these positions the thigh was stabilized using a belt. For the SEATED position, the chair was lifted up at an angle of $90^{\circ}$ from the horizontal and the knee and hip joints were both placed at an angle of $90^{\circ}$.

\section{Instructions}

For each position, MVCs were performed with two different modalities of instructions named ISOL and ALL. In the isolation condition (ISOL), participants were required to produce a force by rotating the footplate as hard as possible and to handle the shoulder harnesses. In this condition, they were invited to use only their calf muscles. In a second condition (ALL), the 
172 participants were invited to grip the ergometer handle and to use all the possible means to create

173 forces against the footplate.

174

175

176

177

178

180

181

182

\section{Estimation of the ankle joint rotation center}

The ankle joint rotation center was estimated using the Symmetrical Centre of Rotation Estimation (SCoRE) method (Ehrig et al. 2006). Briefly, the position of the center of rotation (CR) between two segments is determined by assuming a constant contact point between each and use the relation

$$
C R=\mathbf{o}_{1}+\mathbf{R}_{1} \mathbf{u}=\mathbf{o}_{2}+\mathbf{R}_{2} \mathbf{v}
$$

where $\mathbf{o}_{1}$ and $\mathbf{o}_{2}$ are arbitrary points on segments \#1 (the foot) and \#2 (the leg), $\mathbf{R}_{1}$ and $\mathbf{R}_{2}$ are the rotation matrix transforming the segment coordinate system (SCS) to the global coordinate system and $\mathbf{u}$ and $\mathbf{v}$ are the vector linking respectively $\mathbf{o}_{1}$ and $\mathbf{o}_{2}$ to CR in the foot and leg coordinate system respectively. The SCSs were defined according to Wu et al. (2002). For the estimation of the CR position, participants were seated on the ergometer chair with solely their right foot strapped on the footplate connected to the moment arm of the dynamometer and the ankle joint was moved passively at full but comfortable range of motion for about 10 flexion-extension cycles in order to localize an accurate joint center. The values of $\mathbf{u}$ and $\mathbf{v}$ were then used to estimate the position of the CR relative to the SCSs (foot and leg) in all experimental conditions.

\section{Data analysis}

EMG signals were filtered with a bandpass filter ( $4^{\text {th }}$ order Butterworth) between 20 and $400 \mathrm{~Hz}$. Linear envelopes for each muscle were obtained by low-pass filtering the fully rectified raw EMG signals with a $9 \mathrm{~Hz}$ low-pass filter $\left(2^{\text {nd }}\right.$ order Butterworth, zero lag, (Shiavi et al. 1998). For each condition, the averaged value between $-150 \mathrm{~ms}$ and $150 \mathrm{~ms}$ around the peak 
torque event was extracted (Figure 2) and then normalized by the maximal value obtained over

196 all conditions $\left(=\mathrm{EMG}_{\max }\right)$. These calculations were performed for each muscle and each

197 participant independently.

198 Joint torque and kinematic data were filtered by a $15 \mathrm{~Hz}$ low pass filter ( $2^{\text {nd }}$ order

199 Butterworth filter (Winter 1990)). Joint torque was corrected for gravity by subtracting the 200 baseline, and for each condition the maximal value reached over the three tries given to the 201 participant was extracted for analysis (Figure 2).

\section{Statistics}

Normality of the data has been checked using Shapiro-Wilk's tests. For normally 204 distributed data two-way repeated measure ANOVAs (instruction=ALL and ISOL $\times$ 205 position=SUPINE, PRONE and SEATED) were performed after checking for violations of 206 sphericity using Mauchly's test. Post-hoc analyses were then performed using Bonferroni 207 method (Maxwell 1980). For non-normal distribution non parametric Friedman ANOVAs (one208 way repeated measures ANOVA on ranks) was chosen. Wilcoxon rank sum tests associated 209 with Bonferroni-Dunn corrections were used when the null hypothesis was rejected.

The different biases mentioned in the introduction were rallied in kinematic deviations.

211 They include i) the ankle angle changes (in degrees) during the test due to the compliance of 212 the ergometer moment arm, deformation of the footplate and fasteners compliance; ii) the 213 alignment errors (in $\mathrm{mm}$ ) between the axis of the dynamometer and the functional ankle joint 214 centre of rotation in horizontal and vertical axis during the rest and the MVC. Kinematic 215 deviations were compared to the reference using one-sample Student's t-tests (reference 216 value $=0$ ). A description of the axes is given in Figure 1. 
We assessed the relationships between torque and other variables (i.e., kinematic 218 deviations and EMG activity) using Pearson's correlation coefficient (r). For these analyses, 219 values of each variable and for each participant were converted to z-scores, calculated by 220 subtracting the average (over all conditions) and dividing the result by the SD. Because 221 correlation analysis is very sensitive to the presence of outliers in the data (Chatterjee and Hadi 222 1986), normality of each variable was checked and values of $\mid z$-score $\mid>2.58$ (corresponding to 223 the $99^{\text {th }}$ percentile of the distribution) were discarded from the analysis (Burke 2001). All 224 available data were used ( 3 tries x 3 positions $\mathrm{x} 2$ instructions $\times 16$ subjects).

All statistical analyses were performed with the Statistica ${ }^{\circledR}$ software (Statistica ${ }^{\circledR V 6}$,

226 Statsoft, Maison-Alfort, France). Values are reported as mean \pm SD for normally distributed 227 data and as median [range] instead. A p-value below .05 was considered statistically significant. 


\section{Results}

229

230

231

232

233

234

235

236

237

238

239

240

241

242

243

244

\section{Torque}

The results showed that MVCs were significantly affected by the positions $(F(2,30)=13.2$, $\left.\mathrm{p}<.001, \eta_{\mathrm{p}}{ }^{2}=.60\right)$ and the instructions provided $\left(\mathrm{F}(1,15)=54.7, p<.001, \eta_{\mathrm{p}}{ }^{2}=.80\right.$; Figure 3$)$. Posthoc analyses showed that MVCs were significantly greater in the SUPINE and PRONE positions compared to the SEATED position (pooled data: SUPINE=146.0 \pm 40.5 N.m and PRONE $=145.7 \pm 38.9$ N.m $v s$. SEATED $=118.5 \pm 31.2$ N.m, $p<.001)$. Torque was greater in the ALL condition compared to the ISOL condition for each position $(p<.001)$, corresponding to gains of 43.5 [25.4 170.6]\%, 42.5 [1.4 194.6]\% and 15.3 [9.3 71.9]\% for the SUPINE, PRONE and SEATED position respectively. Gains were significantly lower in SEATED compared to SUPINE $(Z=3.15, p<.001)$ and PRONE $(Z=2.43, p=.015)$ but were similar between SUPINE and PRONE $(\mathrm{Z}=1.55, p=.121)$.

\section{Muscle activation}

EMG variables were not normally distributed. The activity level of TA was greater in ALL compared to ISOL (pooled data: ALL=89.6 [6.5 100]\% vs. ISOL=65.3 [2.7 100]\%, Z=3.21, $p=.001)$ but this effect was present in the SUPINE and PRONE positions only $(\mathrm{Z}=3.31, p<.001$, and $\mathrm{Z}=2.53, p=.011$ respectively). Analysis revealed no main effect of the position on the activity of TA $\left(\chi^{2}=4.75, p=.093\right.$; Figure 4$)$.

Overall positions, Sol activity was greater in ALL compared to ISOL (ALL=85.7 [6.0 100]\%) vs. $\mathrm{ISOL}=66.1[0.7100] \%, \mathrm{Z}=2.84, p=.004)$ but post-hoc analysis revealed significant differences in the SUPINE position only $(\mathrm{Z}=2.43, p=.015)$. There was no main effect of the position on the activity of SOL $\left(\chi^{2}=2.25, p=.325\right)$. 
Activity of GM was greater in ALL compared to ISOL (i.e., pooled data: ALL=88.1 [15.8

$251100] \%$ vs. ISOL $=61.5[1.998 .2] \% ; Z=4.24, p<.001)$. These differences held for the SUPINE and PRONE positions $(\mathrm{Z}=2.84, p=.004$ and $\mathrm{Z}=2.74, p=.006$ respectively) but no differences were found in the SEATED position $(\mathrm{Z}=1.76, p=.08)$. Analysis revealed a main effect of the position $\left(\chi^{2}=17.69, p<.001\right)$ i.e., GM was significantly less activated in the SEATED position (=49.9 $[15.3100] \%$,) compared to the PRONE (=79.4 $\left[\begin{array}{ll}1.9 & 100\end{array} \%\right.$., $\left.Z=3.78, p<.001\right)$ and SUPINE $(=0.83[0.091] \%, Z=3.72, p<.001)$ positions.

ST was maximally activated in the PRONE position in 10 out of 16 participants. The activity of ST was significantly higher in ALL compared to ISOL in SUPINE $(Z=3.46, p<.001)$ and SEATED $(Z=3.00, p=.003)$, but no differences were found in the PRONE position $(Z=0.67$, $p=.502)$. A main effect of the position was found $\left(\chi^{2}=6.94 p=.03116\right)$ i.e., there was higher ST activity in the PRONE position but differences were significant only when compared to the SUPINE's (i.e., pooled data $=74.6[3.6100] \%$ vs. $8.0[0.8100] \%, Z=3.22, p=.001$ ).

VL, RF and Gmax activities possess the same patterns among the experimental conditions and were maximally activated in the SEATED position in most participants i.e., in 15,13 and 15 out-of 16 participants respectively (Figure 4). Friedman ANOVA confirmed the effect of position on VL, RF and Gmax (VL: $\chi^{2}=31.75, p<.001 ; \mathrm{RF}: \chi^{2}=17.44, p<.001$; Gmax: $\chi^{2}=38.31$, $267 p<.001)$. These muscles were significantly more activated in the SEATED compared to the 268 SUPINE and PRONE positions (merged values in SEATED position: VL=97.1 [1.4 100]\%, 269 $\mathrm{RF}=62.5[0.6100] \%$ and $\mathrm{Gmax}=90.7[1.3100] \%) v s . \mathrm{SUPINE}+\mathrm{PRONE}: \mathrm{VL}=6.2[0.390 .5] \%$, $270 \mathrm{RF}=6.8[0.3100] \%$ and $\mathrm{Gmax}=5.1[0.454 .9] \%$, Wilcoxon $\mathrm{Z}$-values ranged from 3.23 to 4.75, $271 \mathrm{p}<.001)$. Analyses indicated that the activity of VL, RF and Gmax were greater in the ALL 272 compared to the ISOL condition in the 3 positions tested (Wilcoxon Z-values and $p$-values 273 ranged from $\mathrm{Z}=2.84, p=.004$ to $\mathrm{Z}=3.51, p<.001$; Figure 4 ). 
275 Kinematic results and statistics are summarized in Table 1. During MVCs the ankle joint angle 276 varied of $-9.73 \pm 4.15^{\circ}$ in average i.e., from $91.0 \pm 5.2^{\circ}$ to $81.3 \pm 4.9^{\circ}$ overall conditions. From 277 rest to $\mathrm{MVC}$ and overall conditions, the $\mathrm{CR}$ varied on $\mathbf{e}_{x}$ of $\Delta \mathrm{X}=+14.8 \pm 8.0 \mathrm{~mm}$ (i.e., from $278 \mathrm{Xrest}=9.7 \pm 22.1 \mathrm{~mm}$ to $\mathrm{XMVC}=24.7 \pm 24.6 \mathrm{~mm}$ ) and of $\Delta \mathrm{Y}=-0.71 \pm 9.6 \mathrm{~mm}$ on $\mathbf{e}_{y}$ (i.e., from 279 Yrest $=3.9 \pm 13.1 \mathrm{~mm}$ to $\mathrm{YMVC}=3.0 \pm 15.0 \mathrm{~mm})$.

280 Correlations

281 All the results on correlation analyses are summarized in Table 2 and indicated that torque was 282 significantly correlated with the activity of the plantar flexors in each position. VL, RF, ST and 283 kinematic variables ( $\Delta \mathrm{Y}$ and the variation in joint angle, $\Delta \theta$ ) were found to be significantly 284 related to torque depending on the position and on the instruction (see Table 2). 


\section{Discussion}

286 The aim of this study was to point out the differences in torque output during maximal voluntary contraction (MVC) in isometric plantarflexion when activating either isolated or global muscle (conditions named ISOL and ALL respectively). The ALL condition was associated with higher EMG activities in most of the recorded muscles, notably in plantarflexor muscles, and was associated with higher joint torque compared to ISOL.

Very large differences were observed between ALL and ISOL, with gains on joint torque of about $40 \%$ in average (Figure 3). Lower torque in seated position could be attributable to muscle mechanics, i.e., force-length relationships (Maganaris 2003), and to impairments in motor units recruitment, as already reported for this particular joint angle configuration (i.e., knee and ankle joint angles set at $90^{\circ}$ of flexion) (Cresswell et al. 1995; Kennedy and Cresswell 2001). In mine with our findings, a previous study showed that plantarflexion torque could be significantly enhanced $(\sim+26 \%)$ in multi-joint compared to isolated plantar flexion (Hahn et al. 2011). However, this study remained inconclusive regarding the differences in EMG activity resulting from these two conditions and used different methodologies to assess joint torque in 300 the multi-joint and isolated contractions, i.e., they used inverse dynamic calculations and 301 ergometer measurements, that proved to provide different results (Herzog 1988; Kaufman et al. 302 1995; Arampatzis et al. 2004). Sasaki et al.(1998) observed an increase in plantarflexion torque 303 linked to jaw clenching, but the conclusions relied on integrated electromyographic activity per 304 unit of time rather than EMG level, and did not check for the influence of mechanical factors, 305 as they focused on jaw clenching only.

306 Interestingly, the value of $40 \%$ found in the present study fits well to differences with that 307 observed in similar studies examining ankle MVC, that is, values ranging from 134 up to 186 
308 N.m, despite similar populations and protocols (Danneskiold-Samsøe et al. 2009; Cresswell et 309 al. 1995; Maganaris 2003). More precisely, considering isometric plantarflexion MVCs in the 310 supine and prone positions, and a population of young male adults, literature reports MVC 311 values ranging from $\sim 134$ N.m [e.g. $142 \pm 42, \mathrm{~N}=10$ (Danneskiold-Samsøe et al. 2009) or 134 $312 \pm 23$ N.m , N=10 (Cresswell et al. 1995)] up to $\sim 186$ N.m [e.g. $172 \pm 15, \mathrm{~N}=8$ (Maganaris $3132003)$ or $186 \pm 28$ N.m $(\mathrm{N}=9)$ (Hahn et al. 2011)]. These differences may pertain to differences 314 in the participants' fitness (i.e., more or less trained participants), but the results suggest that an 315 explanation also bears on the nature of the instructions (ALL vs. ISOL).

\section{Mechanical factors}

317 Misalignment has been shown to induce bias of $\sim 10 \%$ in the estimation of joint torque 318 (Arampatzis et al. 2007; Deslandes et al. 2008), but this factor is not expected to create large 319 differences among studies, as the positioning of the foot is expected to be carefully executed

320 Given the equation \#1, positive deviations of CR in the x-direction, that decreases $\frac{r_{\text {ankle }}}{r_{\text {dynamometer }}}$,

321 decreases the effectiveness of the ankle torque. The misalignments observed in this study on 322 the $\mathrm{X}$-axis are positive and then, they are not likely to explain the gains in torque. Nevertheless, 323 misalignments may allow the auxiliary muscles, through joint reaction forces transmission, to 324 influence the ankle torque.

325 One can first observe that positioning have an effect on the activity of knee extensors, knee 326 flexors and hip extensors muscles (Figure 4). For example, the seated position was associated 327 with higher level of activity for VL, RF and Gmax and the prone position was associated with 328 higher ST activity (Figure 4) but the correlation values between these muscles and the torque 329 produced remained modest (Table 2), suggesting that the mechanical influence of these muscles 330 is small. Furthermore, despite the fact that differences were observed in ST activity between 
331 PRONE and SUPINE, no differences were found in torque between these two positions.

332 Additionally, the large increase in Gmax, VL and RF activity (Figure 4) did not preclude to the

333 force deficit associated with the seated position (Figure 3). As a consequence, no major

334 mechanical influence of these muscles is expected on the produced torque. Notwithstanding, at

335 least two observations forbid ruling out the influence of such forces. Firstly, despite significant

336 and positive correlations of the activity of Sol and GM with torque in the seated position (Table

337 2), the increase in torque was not associated with significant increases in the activity of these

338 muscles i.e., SOL $(p=.255)$ and $\mathrm{GM}(p=.079)$. And in this particular position, VL, RF and ST

339 were also correlated with torque (Table 2). These observations strongly suggest that the forces

340 created by muscles that do not span over the ankle joint significantly influenced the measured

341 joint torque, at least in the seated position. Secondly, provided that torque is mainly related to

342 plantarflexor activity, as the relation between EMG and torque tends to be convex toward 343 tension at high force levels (Perry and Bekey 1981; Lawrence and De Luca 1983), a given

344 increase in torque in this portion of the curve should have been associated with a larger increase

345 in EMG and not with a similar one (Figure 3 and Figure 4). Suggesting that even in the supine

346 and prone positions, plantarflexors are not the sole contributors of the increase in torque.

\section{$347 \quad$ Neural factors}

348 What can explain the higher muscle activity level in ALL compared to ISOL observed in this 349 study? First, motivation is not likely to explain the differences observed between ALL and 350 ISOL. Although motivation has not been explicitly assessed in this study, differences in 351 motivation are not expected, because the tests were randomized. Additionally, we found high 352 reliability between the 3 trials within all the conditions tested, with an average correlation 353 coefficient of .95 (range=[.90 .98]; model corresponding to case \#1 in McGraw et al. (1996)) 354 and an average coefficient of variation of $5.8 \%$ (range $=\left[\begin{array}{ll}3.2 & 8.2\end{array}\right]$ ). These reliability values are 
highly consistent with previous reports testing MVCs in plantarflexion (Webber and Porter

356 2010; Todd et al. 2004; Sleivert and Wenger 1994) and can be taken as evidences that MVC

357 testing conditions carried out here can be truly compared to those imposed in previous studies.

358 As a consequence, the lower values observed in ISOL are not likely to be due to a lack of

359 motivation from the participants.

360 In ALL, participants were allowed to grasp the ergometer, which is not generally allowed in

361 studies measuring ankle MVCs (cf. a representative setup in Figure \#1 of Simoneau et al,

362 (2009), so that concurrent activation potentiation could be induced (Ebben et al. 2008a; Ebben

363 et al. 2010; Cherry et al. 2010; Ebben et al. 2008b). Jaw clenching or Valsalva maneuver have

364 been reported to improve the level of maximal activation of the contracting muscles (Ebben et

365 al. 2008b; Sasaki et al. 1998). However, most of these studies focused on the knee extensors

366 muscles (Ebben et al. 2008b; Ebben et al. 2010), and not on plantarflexors. Furthermore,

367 improvements in peak torque due to jaw clenching and Valsalva maneuver have been reported

368 to be of $\sim 15 \%$ for the quadriceps muscles (Ebben et al. 2008b; Ebben et al. 2010), that is, far

369 less than the differences observed in the present study (i.e., $\sim 40 \%$ ). This may suggest a

370 particular sensitivity of the plantarflexors to the phenomenon. In fact, contrary to other muscles

371 such as elbow flexors (Herbert et al. 1998) or ankle dorsiflexors (Kent-Braun et al. 2002),

372 activation of plantarflexor muscles is rarely complete (Todd et al. 2004; Cresswell et al. 1995).

373 Without training or adequate testing conditions, plantarflexor muscles are not maximally

374 activated by volition, and only reach about $80-90 \%$ of voluntary activation (Cresswell et al.

375 1995; Maffiuletti et al. 2002). This is in line with the finding that the neural drive to these

376 muscles can be significantly improved by a strength training (Shield and Zhou 2004) or

377 imagined strength training (Zijdewind et al. 2003; Sidaway and Trzaska 2005), whereas such

378 is not the case for the elbow flexors, for example which possess a high initial level of voluntary 
379 activation (Herbert et al. 1998). The work of Devanne and collaborators (Devanne et al. 2002;

380 Kouchtir-Devanne et al. 2012) is particularly interesting in this respect. They observed that the 381 excitability of the cortical neurons associated with the first dorsal interosseus was lower during 382 isolated (contraction of first dorsal interosseus only) vs. global muscle contractions (precision 383 grip implying the thumb and the finger). This indicates that the cortical excitability of a given 384 muscle depends on its functional interconnections at the cortical level. These findings support 385 the idea that isolated contractions, which explicitly or implicitly (through instructions) require 386 a selection of the contracting muscles, may induce inhibition, incompatible with the objectives 387 of MVC testing. Allowing global muscle activation or not is then a critical aspect of the 388 instructions

389 Conclusions

390 This study reports that activation of plantarflexor muscles are superior during global muscle 391 activation compared to isolated joint contraction, entailing very large differences in motor392 output. It emphasizes the pertinence of using isolated vs. unconstrained MVC testing protocols, 393 notably for muscles that are not maximally activated by volition. 
Arampatzis A, De Monte G, Morey-Klapsing G (2007) Effect of contraction form and contraction velocity on the differences between resultant and measured ankle joint moments. Journal of biomechanics 40 (7):1622-1628

Arampatzis A, Karamanidis K, De Monte G, Stafilidis S, Morey-Klapsing G, Brüggemann GP (2004) Differences between measured and resultant joint moments during voluntary and artificially elicited isometric knee extension contractions. Clinical biomechanics 19 (3):277-283

Barry B, Riley Z, Pascoe M, Enoka R (2008) A spinal pathway between synergists can modulate activity in human elbow flexor muscles. Experimental Brain Research 190 (3):347-359

Burke S (2001) Missing values, outliers, robust statistics \& non-parametric methods. LC-GC Europe Online Supplement, Statistics \& Data Analysis 2:19-24

Chatterjee S, Hadi AS (1986) Influential observations, high leverage points, and outliers in linear regression. Statistical Science 1 (3):379-393

Cherry EA, Brown LE, Coburn JW, Noffal GJ (2010) Effect of remote voluntary contractions on knee extensor torque and rate of velocity development. Journal of Strength and Conditioning Research 24 (9):2564-2569

Cresswell AG, Löscher W, Thorstensson A (1995) Influence of gastrocnemius muscle length on triceps surae torque development and electromyographic activity in man. Experimental brain research 105 (2):283-290

Danneskiold-Samsøe B, Bartels E, Bülow P, Lund H, Stockmarr A, Holm C, Wätjen I, Appleyard M, Bliddal H (2009) Isokinetic and isometric muscle strength in a healthy population with special reference to age and gender. Acta Physiologica 197 (s673):1-68

De Ruiter CJ, Hoddenbach JG, Huurnink A, De Haan A (2008) Relative torque contribution of vastus medialis muscle at different knee angles. Acta Physiologica 194 (3):223-237.

Deslandes S, Mariot J-P, Serveto S (2008) Offset of rotation centers creates a bias in isokinetics: A virtual model including stiffness or friction. Journal of biomechanics 41 (10):21122120.

Devanne H, Cohen LG, Kouchtir-Devanne N, Capaday C (2002) Integrated Motor Cortical Control of Task-Related Muscles During Pointing in Humans. Journal of Neurophysiology 87 (6):3006-3017

Drouin JM, Valovich-mcLeod TC, Shultz SJ, Gansneder BM, Perrin DH (2004) Reliability and validity of the Biodex system 3 pro isokinetic dynamometer velocity, torque and position measurements. European journal of applied physiology 91 (1):22-29

Ebben WP (2006) A brief review of concurrent activation potentiation: theoretical and practical constructs. Journal of Strength and Conditioning Research 20 (4):985-991.

Ebben WP, Flanagan EP, Jensen RL (2008a) Jaw clenching results in concurrent activation potentiation during the countermovement jump. Journal of Strength and Conditioning Research 22 (6):1850-1854.

Ebben WP, Leigh DH, Geiser CF (2008b) The effect of remote voluntary contractions on knee extensor torque. Medicine \& Science in Sports \& Exercise 40 (10):1805-1809.

Ebben WP, Petushek EJ, Fauth ML, Garceau LR (2010) EMG analysis of concurrent activation potentiation. Medicine \& Science in Sports \& Exercise 42 (3):556-562.

Ehrig RM, Taylor WR, Duda GN, Heller MO (2006) A survey of formal methods for determining the centre of rotation of ball joints. Journal of biomechanics 39 (15):27982809 
Gandevia SC (2001) Spinal and Supraspinal Factors in Human Muscle Fatigue. Physiological Reviews 81 (4):1725-1789

Hahn D, Olvermann M, Richtberg J, Seiberl W, Schwirtz A (2011) Knee and ankle joint torqueangle relationships of multi-joint leg extension. Journal of biomechanics 44 (11):20592065

Herbert R, Dean C, Gandevia S (1998) Effects of real and imagined training on voluntary muscle activation during maximal isometric contractions. Acta Physiologica Scandinavica 163 (4):361-368

Hermens HJ, Freriks B, Disselhorst-Klug C, Rau G (2000) Development of recommendations for SEMG sensors and sensor placement procedures. Journal of Electromyography and Kinesiology 10 (5):361-374

Herzog W (1988) The relation between the resultant moments at a joint and the moments measured by an isokinetic dynamometer. Journal of biomechanics 21 (1):5-12

Hicks J (1953) The mechanics of the foot: I. The joints. Journal of Anatomy 87 (Pt 4):345

Horak FB, Shupert CL, Mirka A (1989) Components of postural dyscontrol in the elderly: a review. Neurobiology of aging 10 (6):727-738

Kaufman KR, An K-N, Chao E (1995) A comparison of intersegmental joint dynamics to isokinetic dynamometer measurements. Journal of biomechanics 28 (10):1243-1256

Kennedy P, Cresswell A (2001) The effect of muscle length on motor-unit recruitment during isometric plantar flexion in humans. Experimental brain research 137 (1):58-64

Kent-Braun JA, Ng AV, Doyle JW, Towse TF (2002) Human skeletal muscle responses vary with age and gender during fatigue due to incremental isometric exercise. Journal of Applied Physiology 93 (5):1813-1823

Klass M, Baudry S, Duchateau J (2008) Age-related decline in rate of torque development is accompanied by lower maximal motor unit discharge frequency during fast contractions. Journal of Applied Physiology 104 (3):739-746

Kouchtir-Devanne N, Capaday C, Cassim Fo, Derambure P, Devanne H (2012) Task-dependent changes of motor cortical network excitability during precision grip compared to isolated finger contraction. Journal of Neurophysiology 107 (5):1522-1529.

Lawrence JH, De Luca C (1983) Myoelectric signal versus force relationship in different human muscles. Journal of Applied Physiology 54 (6):1653-1659

Lundberg A, Svensson O, Nemeth G, Selvik G (1989) The axis of rotation of the ankle joint. Journal of Bone \& Joint Surgery, British Volume 71 (1):94-99

Maffiuletti NA, Pensini M, Martin A (2002) Activation of human plantar flexor muscles increases after electromyostimulation training. Journal of Applied Physiology 92 (4):1383-1392

Maganaris CN (2003) Force-length characteristics of the in vivo human gastrocnemius muscle. Clinical anatomy $16(3): 215-223$

Maxwell SE (1980) Pairwise multiple comparisons in repeated measures designs. Journal of Educational and Behavioral Statistics 5 (3):269-287

McGraw KO, Wong S (1996) Forming inferences about some intraclass correlation coefficients. Psychological methods 1 (1):30

McNeil CJ, Vandervoort AA, Rice CL (2007) Peripheral impairments cause a progressive agerelated loss of strength and velocity-dependent power in the dorsiflexors. Journal of Applied Physiology 102 (5):1962-1968

Moraux A, Canal A, Ollivier G, Ledoux I, Doppler V, Payan C, Hogrel J-Y (2013) Ankle dorsiand plantar-flexion torques measured by dynamometry in healthy subjects from 5 to 80 years. BMC musculoskeletal disorders 14 (1):104 
Perry J, Bekey GA (1981) EMG-force relationships in skeletal muscle. Critical Reviews in Biomedical Engineering 7 (1):1-22

Ramos MU, Knapik J (1978) Instrumentation and techniques for the measurement of muscular strength and endurance in the human body. DTIC Document

Sasaki Y, Ueno T, Taniguchi H, Ohyama T (1998) Effect of teeth clenching on isometric and isokinetic strength of ankle plantar flexion. Journal of medical and dental sciences 45 (1):29

Shiavi R, Frigo C, Pedotti A (1998) Electromyographic signals during gait: criteria for envelope filtering and number of strides. Med Biol Eng Comput 36 (2):171-178

Shield A, Zhou S (2004) Assessing Voluntary Muscle Activation with the Twitch Interpolation Technique. Sports Medicine 34 (4):253-267.

Sidaway B, Trzaska A (2005) Can Mental Practice Increase Ankle Dorsiflexor Torque? Physical therapy 85 (10):1053-1060

Simoneau E, Martin A, Van Hoecke J (2007) Effects of joint angle and age on ankle dorsi-and plantar-flexor strength. Journal of Electromyography and Kinesiology 17 (3):307-316

Simoneau EM, Billot M, Martin A, Van Hoecke J (2009) Antagonist mechanical contribution to resultant maximal torque at the ankle joint in young and older men. Journal of Electromyography and Kinesiology 19 (2):e123-e131

Sleivert GG, Wenger HA (1994) Reliability of measuring isometric and isokinetic peak torque, rate of torque development, integrated electromyography, and tibial nerve conduction velocity. Archives of physical medicine and rehabilitation 75 (12):1315-1321

Todd G, Gorman RB, Gandevia SC (2004) Measurement and reproducibility of strength and voluntary activation of lower limb muscles. Muscle \& nerve 29 (6):834-842

Van Cutsem M, Duchateau J, Hainaut K (1998) Changes in single motor unit behaviour contribute to the increase in contraction speed after dynamic training in humans. The Journal of Physiology 513 (1):295-305

Webber SC, Porter MM (2010) Reliability of ankle isometric, isotonic, and isokinetic strength and power testing in older women. Physical therapy 90 (8):1165-1175

Winter DA (1990) Biomechanics and motor control of human movement. Wiley, New York

Wu G, Siegler S, Allard P, Kirtley C, Leardini A, Rosenbaum D, Whittle M, D'Lima DD, Cristofolini L, Witte H (2002) ISB recommendation on definitions of joint coordinate system of various joints for the reporting of human joint motion-part I: ankle, hip, and spine. Journal of biomechanics 35 (4):543-548

Zijdewind I, Toering ST, Bessem B, van der Laan O, Diercks RL (2003) Effects of imagery motor training on torque production of ankle plantar flexor muscles. Muscle \& Nerve 28 (2):168-173 


\begin{tabular}{|c|c|c|c|c|c|c|}
\hline & \multicolumn{2}{|c|}{ SUPINE } & \multicolumn{2}{|c|}{ PRONE } & \multicolumn{2}{|c|}{ SEATED } \\
\hline & ALL & ISOL & ALL & ISOL & ALL & ISOL \\
\hline $\mathrm{X}$ rest & $22,5 \pm 13,5$ & $20,9 \pm 14,6$ & $-14,8 \pm 11,3$ & $-14,7 \pm 16,0$ & $20,0 \pm 15,3$ & $22,1 \pm 16,4$ \\
\hline Y rest & $3,9 \pm 9,7$ & $4,4 \pm 10,8$ & $-5,2 \pm 12,8$ & $-6,0 \pm 11,3$ & $12,4 \pm 10,4$ & $15,4 \pm 9,7$ \\
\hline X MVC & $39,3 \pm 14,1$ & $36,1 \pm 13,0$ & $-2,6 \pm 13,6$ & $-4,4 \pm 17,0$ & $41,0 \pm 18,0$ & $36,2 \pm 15,4$ \\
\hline Y MVC & $-0,7 \pm 10,3$ & $11,6 \pm 11,8$ & $-12,5 \pm 12,5$ & $1,5 \pm 10,1$ & $1,9 \pm 14,1$ & $18,1 \pm 12,1$ \\
\hline$\Delta \mathrm{X}$ & $16,9 \pm 5,8$ & $15,2 \pm 7,3$ & $12,2 \pm 12,7$ & $9,8 \pm 4,9$ & $21,0 \pm 5,3$ & $14,1 \pm 4,9$ \\
\hline$\Delta \mathrm{Y}$ & $-4,6 \pm 4,7$ & $7,3 \pm 3,5$ & $-7,1 \pm 8,1$ & $\mathbf{7 , 5} \pm \mathbf{7 , 5}$ & $-10,6 \pm 9,3$ & $2,7 \pm 5,4$ \\
\hline$\Delta \theta$ ankle & $-6,71 \pm 3,81$ & $-7,70 \pm 2,67$ & $-9,51 \pm 3,32$ & $-9,63 \pm 4,80$ & $-14,29 \pm 3,14$ & $-10,70 \pm 2,51$ \\
\hline
\end{tabular}

527

528

529

530

531

532

533

Table 1. Kinematic variables. $\mathrm{X}$ and $\mathrm{Y}$ are the position of the ankle joint (estimated by the $\mathrm{SCoRe}$ method) relative to the axis of rotation of the dynamometer in the X-direction and $\mathrm{y}$ direction at rest (X rest and $\mathrm{Y}$ rest) and at the peak torque event (X MVC and. Y MVC), given in $\mathrm{mm} . \Delta \mathrm{X}=\mathrm{X}$ MVC- $\mathrm{X}$ rest, and $\Delta \mathrm{Y}=\mathrm{Y}$ MVC- $\mathrm{Y}$ rest. $\Delta \theta$ is the difference in joint angle in degree between MVC and rest, in degree. Bolded values indicate a significant difference from 0 (t-test for single mean; $p<.05$ ). Values are given as mean $\pm \mathrm{SD}$.

\begin{tabular}{|c|c|c|c|c|c|c|}
\hline \multirow{3}{*}{$\begin{array}{c}\text { torque vs. } \\
\text { TA }\end{array}$} & \multicolumn{2}{|c|}{ SUPINE } & \multicolumn{2}{|c|}{ PRONE } & \multicolumn{2}{|c|}{ SEATED } \\
\hline & ISOL & ALL & ISOL & ALL & ISOL & ALL \\
\hline & 0.10 & $0.55 * *$ & 0.29 & $0.67 * *$ & 0.29 & 0.10 \\
\hline Sol & -0.11 & $0.42 * *$ & $0.41 *$ & $0.64 * *$ & $0.59 * *$ & 0.18 \\
\hline GM & $0.40 *$ & 0.16 & 0.30 & $0.57 * *$ & $0.42 *$ & $0.55 * *$ \\
\hline VL & -0.28 & -0.11 & 0.22 & 0.25 & 0.38 * & $0.35 *$ \\
\hline RF & -0.24 & -0.10 & 0.19 & $0.34 *$ & 0.24 & 0.16 \\
\hline ST & $0.31 *$ & 0.16 & 0.17 & 0.00 & $0.54 * *$ & 0.27 \\
\hline Gmax & -0.29 & -0.19 & 0.22 & 0.19 & 0.16 & 0.04 \\
\hline$\Delta \mathbf{X}$ & 0.17 & 0.01 & 0.23 & 0.03 & 0.27 & 0.24 \\
\hline$\Delta \mathbf{Y}$ & $0.30 *$ & -0.17 & -0.08 & -0.24 & -0.06 & -0.29 \\
\hline$\Delta \theta$ & -0.07 & 0.21 & -0.28 & -0.21 & -0.26 & $-0.37 *$ \\
\hline $\mathbf{N}$ & 46 & 47 & 36 & 37 & 42 & 37 \\
\hline
\end{tabular}

Table 2. Correlation coefficients. $\mathrm{N}$ refers to the number of values used to compute the Pearson's r. Bolded values indicate significant correlations $\left({ }^{*}: \mathrm{p}<.05 ; * *: \mathrm{p}<0.001\right)$. 

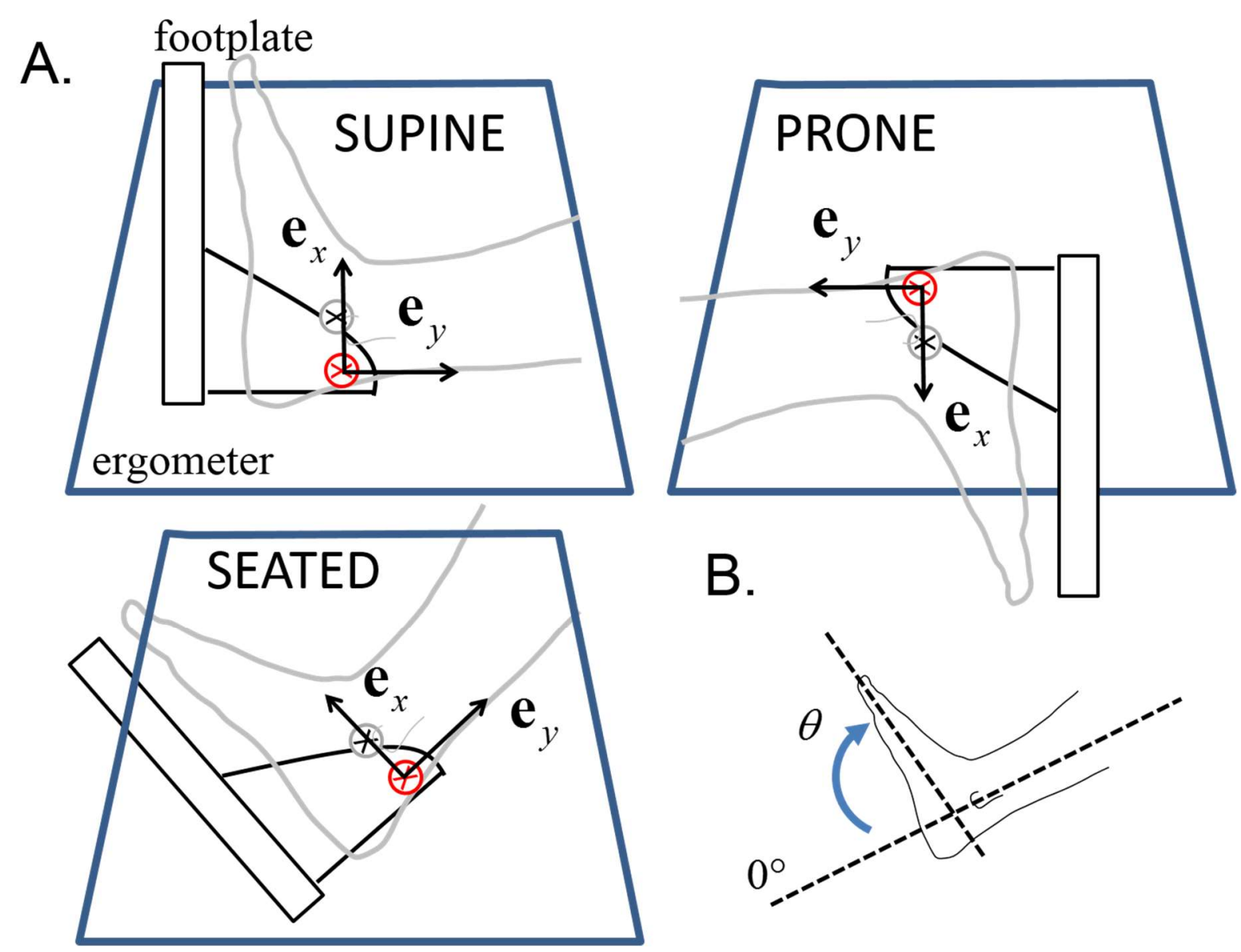

B.

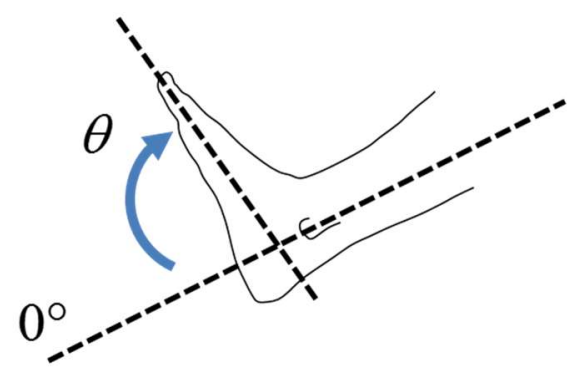

540 Figure 1. Position of the foot relative to the ergometer. A. The circled red cross designs the ergometer axis of rotation and the circled black cross the ankle axis of rotation $(=\mathrm{CR})$. In all positions, the $\mathrm{X}$-axis associated with the vector $\mathbf{e}_{x}$ is the axis parallel to the footplate and pointing toward the participant toes and the Y-axis asociated with the vector $\mathbf{e}_{y}$ is the axis orthogonal to it and pointing toward the participant leg. The origin is centered at the level of 


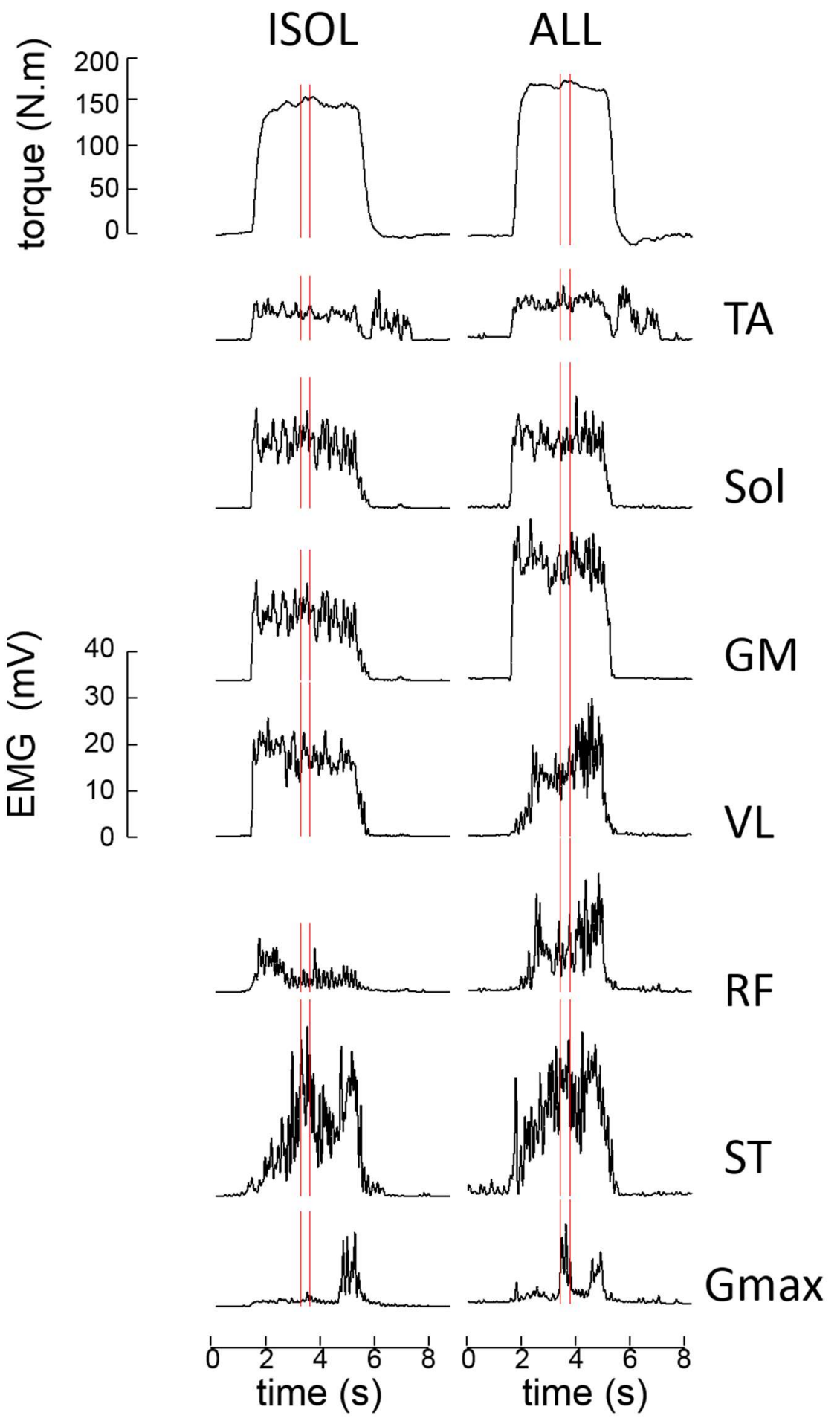

551 Figure 2. Example of torque and EMG data for a typical participant. Condition = supine position. Smoothed torque and EMG envelope are processed as indicated in section Data analysis. Vertical lines indicate the region around peak torque used for analysis.: tibialis anterior. SOL: soleus. GM: gastrocnemius medialis $\mathbf{V L}$ : vastus lateralis. RF: rectus femoris.

555 ST: semi tendinosus. Gmax: gluteus maximus. 


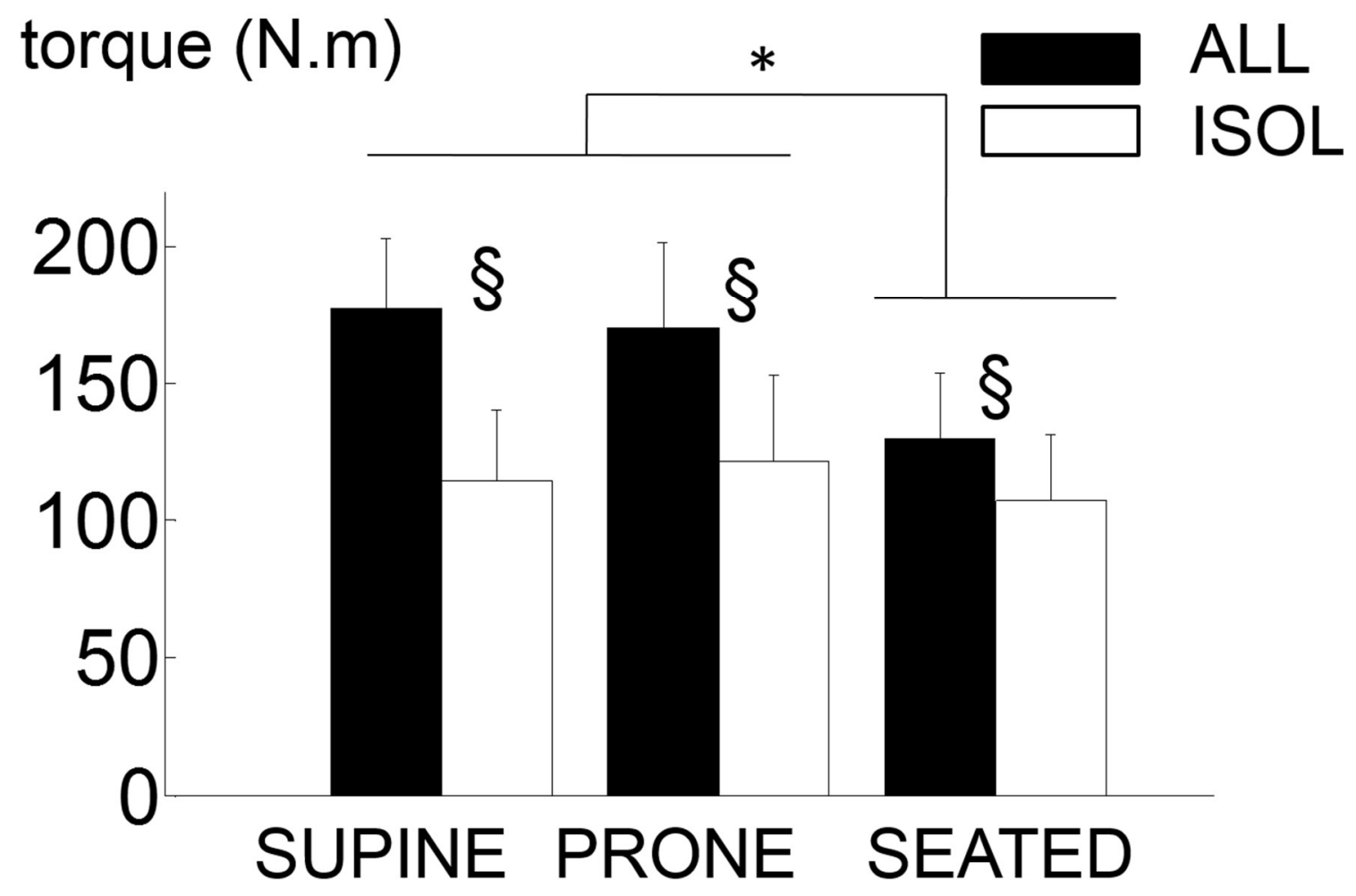

557 Figure 3. Torque data. Bars represent the mean and error-bars one SD. $\S$ indicates a significant 558 difference $(\mathrm{p}<.05)$ between ALL and ISOL. * indicates a significant difference between 559 [SUPINE and PRONE] $v$. SEATED $(p<.05)$. See section RESULTS for details. 
TA

a.u. 0.6
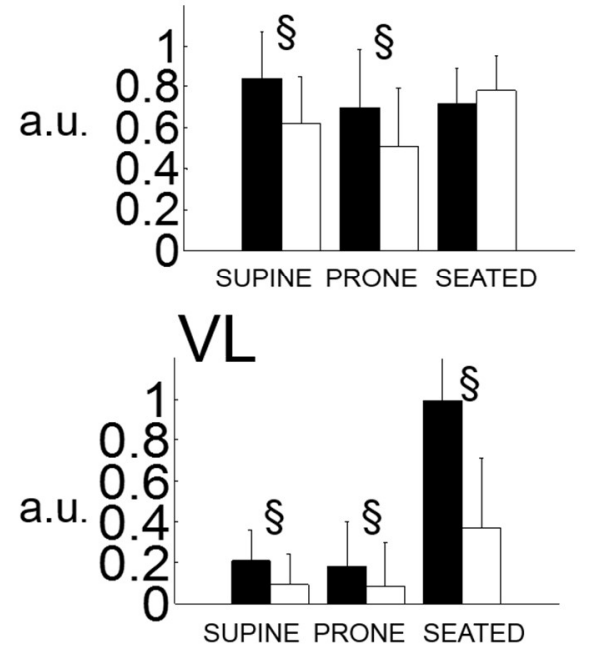

Gmax

a.u.

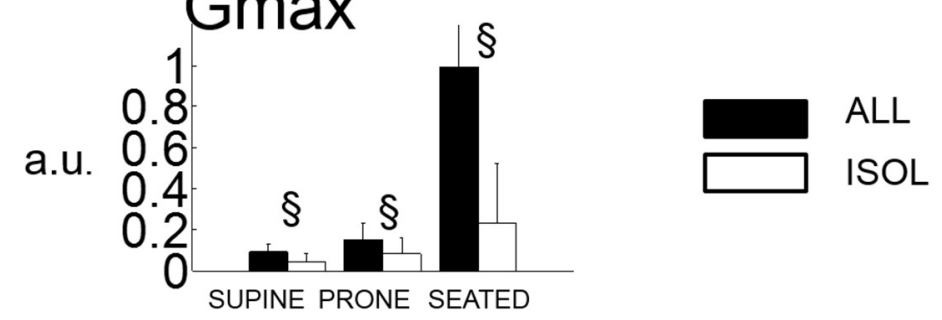

Sol

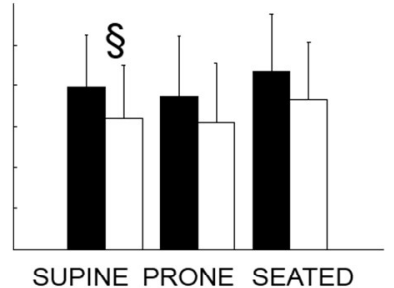

RF

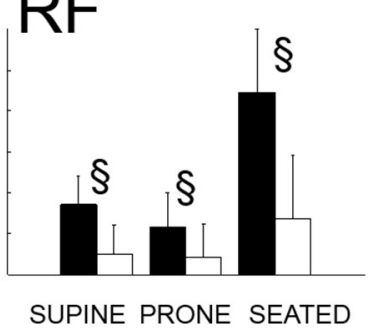

GM

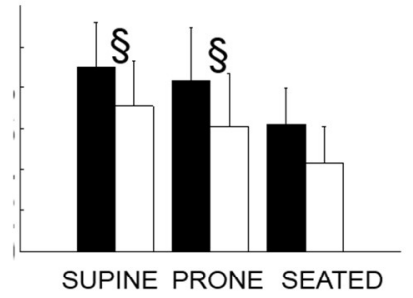

ST

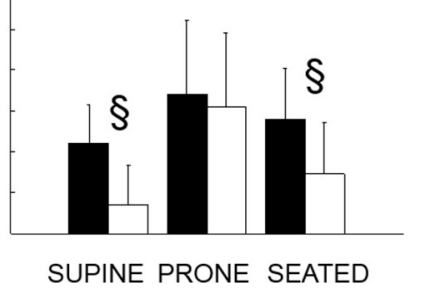

563 Figure 4. Normalized EMG activities. Bars represent the mean and error-bars one SD. $\S$ 564 indicates a significant difference $(p<.05)$ between ALL and ISOL. 